\title{
MALEZAS ASOCIADAS A CANALES DE RIEGO Y TERRENOS COLINDANTES DE ARROZ ANEGADO EN FINCA EL CERRITO, GUANACASTE, COSTA RICA ${ }^{1}$
}

\author{
Manuel Rojas ${ }^{2}$, Renán Agüero ${ }^{3}$
}

\section{RESUMEN}

Malezas asociadas a canales de riego y terrenos colindantes de arroz anegado en Finca El Cerrito, Guanacaste, Costa Rica. Se realizó un inventario de malezas en canales de riego y zonas aledañas dedicadas al cultivo del arroz anegado, en la Finca El Cerrito, Liberia, Provincia de Guanacaste, Costa Rica. El muestreo se hizo en las dos épocas climáticas durante 1992. Cada punto de muestreo fue inventariado en cinco hábitats ( Berna, Talud interior, Talud exterior, Plan y Zona de cultivo). En cada hábitat se registraron las especies presentes, su grado de cobertura y su fenología. Para cada especie se calculó un Indice de FrecuenciaCobertura (IFC) y un Indice de Participación Relativa (IPR). También se calculó el IPR de cada familia de malezas. Se identificaron 131 especies en la época seca y 144 en la lluviosa. Esto indica una gran diversidad asociada a los canales de riego. La mayor cantidad de especies se localizaron en la berna y los taludes, la menor cantidad de especies se registró en la zona del cultivo. La familia más importante de acuerdo al IPR fue Poaceae, le siguió Cyperaceae. Echinochloa colonum mostró IFC altos y apareció en todos los hábitat. Esta especie y otras como Oryza rufipogon, O. latifolia, Cyperus iria y varias latifoliadas, a pesar de ser controladas dentro del cultivo, se reproducen con facilidad en las estructuras del canal y sus semillas son introducidas de nuevo al cultivo con el agua de riego.

\begin{abstract}
A survey of weeds associated to irrigation canals and adjacent inundated rice fields at Finca El Cerrito, Guanacaste, Costa Rica. A weed survey was conducted on irrigation canals and neighboring inundated rice fields, at Finca El Cerrito, Liberia, Guanacaste, Costa Rica. Sampling was performed on two growing seasons contrasting in weather conditions (rainy and dry) during 1992. Each surveying point was subdivided in five habitats (levee, internal slope, external slope, bottom of canal, and neighboring rice field). Plant species, percent cover and phenological stage were recorded at each habitat. In addition, for each species the next parameters were estimated: Frecuency-Cover Index (IFC), and Relative Participation Index (IPR). IPR at the family level was also calculated. 131 species during the dry season, and 144 species during the rainy season were recorded, showing that there is a significant plant diversity associated to these irrigation canals. The levee showed the greatest amount of plant species, while crop fields the least. Poaceae was the most important family, followed by Cyperaceae, based on IPR. Echinochloa colonum showed high IFC values and appeared in all habitats; this species together with others such as Oryza rufipogon, $O$. latifolia, Cyperus iria, and several broadleaf weeds are carefully controlled in rice fields, but reproduce heavily on irrigation canals, and shed their seeds in irrigation water, thus reinfesting commercial fields.
\end{abstract}

\section{INTRODUCCION}

El riego no sólo se limita al uso del agua, sino que involucra un cambio tecnológico, lo cual también va asociado a un cambio en el agroecosistema. Ambos aspectos tecnología y agroecosistemas plantean nuevos retos al productor. Es necesario conocer con detalle las alteraciones producidas en el agroecosistema con la introducción del riego para así responder mejor a los nuevos desafíos. Uno de los componentes que más rápido responde a variaciones en el ambiente por el riego es la vegetación, porque se les facilita un medio de diseminación eficaz y se les suple del factor que las limita en la época seca.

La composición florística y la abundancia relativa de las malezas son importantes componentes del manejo integrado de malezas en agroecosistemas (Thomas

\footnotetext{
Presentado en la XLI Reunión Anual del PCCMCA en Honduras, América Central. 26 de marzo - 1 de abril, 1995.

2 Proyecto financiado por PRIAG. Standard Fruit Co. Valle de la Estrella, Costa Rica.

3 Centro de Investigaciones Agronómicas. Universidad de Costa Rica.
} 
1991). Esta información es necesaria para desarrollar una estrategia efectiva de combate de malezas. La frecuencia, el área infestada y la densidad de la población de malezas puede obtenerse sólo mediante estudios cuantitativos. Estos permitirán además establecer variaciones en la población debido a cambios en el patrón de uso de herbicidas y prácticas culturales, siempre y cuando se cuente con cierta información base que se pueda usar para comparar los resultados de futuras investigaciones.

Con el proyecto de Riego Arenal-Tempisque, en la provincia de Guanacaste, Costa Rica, se pretende abarcar un área de riego de 66.000 hectáreas. Un estudio detallado de la comunidad de malezas asociadas a los canales de riego es fundamental para establecer las especies dominantes, y anticipar problemas; sin embargo esto no se ha hecho. Uno de los pocos estudios fitosociológicos realizados en esta provincia fue el de Sánchez (1993), en campos de melón y áreas colindantes a este cultivo, con el objetivo de identificar las especies hospederas de virus de este cultivo, por lo que se excluyeron de los levantamientos a priori gramíneas y ciperáceas.

Por la importancia de este distrito de riego, el Centro de Investigación en Protección de Cultivos (CIPROC), de la Universidad de Costa Rica, desarrolló un programa de investigación de malezas, en arroz bajo aniego, en esta región. El presente estudio forma parte de dicho proyecto de investigación. El objetivo principal fue generar información básica sobre las principales malezas asociadas a los canales de riego y al cultivo de arroz bajo aniego en el Distrito de Riego Arenal-Tempisque.

\section{MATERIALES Y METODOS}

El levantamiento se realizó en la finca El Cerrito, en el cantón de Liberia, Provincia de Guanacaste, Costa Rica.

Las unidades de muestreo se escogieron siguiendo la metodología propuesta por Matteucci y Colma (1982). Se usó un mapa de la zona de estudio y se seleccionó al azar los canales. El área de muestreo, se determinó según la metodología propuesta por Braun-Blanquet (1979).

En cada canal de riego, se hizo un submuestreo en los siguientes hábitats: berna, talud interior, talud exterior, plan y zona de cultivo.

La identificación de las especies fue hecha en colaboración con taxónomos vegetales del Herbario
Nacional, Universidad de Costa Rica, y Universidad Nacional.

La cobertura relativa de cada especie se obtuvo por estimación visual (Cuadro 1).

Cuadro 1. Escala de cobertura relativa para el estudio de la población de malezas.

\begin{tabular}{cll}
\hline Valor & Porcentaje de cobertura & Marca de clase* \\
\hline 0 & Ausente & 0 \\
1 & Menor del 1\% & 0,5 \\
2 & $1 \%-5 \%$ & 3,5 \\
3 & $6 \%-30 \%$ & 18,5 \\
4 & $31 \%-66 \%$ & 49 \\
5 & $67 \%-100 \%$ & 84,0 \\
\hline
\end{tabular}

* La marca de clase es el valor medio del intervalo porcentual de cada categoría.

Fuente: Soto y Agüero, 1992.

Se determinó la frecuencia relativa, hábitat (presencia de la especie en cualquiera de los hábitats anteriormente señalados), época de crecimiento (época seca y lluviosa), fenología. La fenología se determinó con la codificación propuesta por Soto y Agüero (1992). Con la frecuencia y la cobertura relativas se calcularon los siguientes índices:

a- Indice de frecuencia-cobertura (IFC) por hábitat:

$\mathrm{IFC}=\mathrm{F} * \mathrm{Mc}$ (fórmula 1)

donde: $\quad$ IFC $=$ Indice de Frecuencia-Cobertura

$\mathrm{F}=$ Frecuencia relativa

Mc = Marca de clase de la cobertura relativa

b- Indice de frecuencia-cobertura general:

IFC general $=$ IFC berna + IFC t. interior + IFC t. exterior + IFC plan + IFC cultivo

(fórmula 2)

c- Indice de frecuencia-cobertura familiar:

Correspondió a la suma de los IFC generales de las especies de una familia.

d- Indice de participación relativa (IPR):

IPR = IFC familia/ Suma de todos los IFC

(fórmula 3)

Permite establecer la importancia de las familias en la comunidad vegetal. 


\section{RESULTADOS Y DISCUSION}

En la época seca se identificaron 131 especies y en la lluviosa 144 (Cuadro 2).

Se dio un incremento en el IFC al pasar de la época seca a la lluviosa. Sin embargo, las familias Aizoaceae, Alismataceae, Asclepiadaceae, Boraginaceae, Combretaceae, Gentianaceae, Hidrocharitaceae, Labiatae, Marsileaceae, Molluginaceae, Nymphaeaceae, Passifloraceae, Salvinaceae, Sapindaceae, Sterculiaceae, y Vitaceae, redujeron su IPR al pasar de la época seca a la lluviosa. La mayor parte de estas familias estuvieron representadas por una especie, por lo que las variaciones sufridas por la especie afectaron los valores de la familia. Lo anterior hace suponer que la mayor luminosidad y/o menor humedad relativa de la época seca podría favorecerlas. Se deben hacer estudios más detallados al respecto, dado que todas estas familias tuvieron IPR bajos.

Determinar la época en que se presenta una mayor cobertura de una o varias especies puede ser importante para definir estrategias de manejo. Así, especies que presentaron niveles muy bajos en la época seca y que después aumentaron en la época lluviosa, deben ser sometidas a un programa de manejo desde la transición entre ambas épocas o al inicio de las lluvias. La mayor atención desde el punto de vista de combate debe ser puesto sobre las poáceas y las ciperáceas en las dos épocas del año, ya que son familias que contienen especies de importancia en el cultivo. Controladas éstas, se podría fomentar una flora diversa no dañina en las bernas.

La tercera familia en importancia fue la Hidrocharitaceae, con una sola especie Hydrilla verticillata, a

Cuadro 2. Indice de frecuencia-cobertura (IFC) para cada especie y familia e indice de participación relativa (IPR) familiar. Finca El Cerrito, Liberia. Guanacaste. Epocas seca y lluviosa. 1992.

\begin{tabular}{|c|c|c|c|c|c|c|c|}
\hline \multirow[t]{3}{*}{ Familia } & \multirow[t]{3}{*}{ Especie } & \multicolumn{3}{|c|}{ Epoca seca } & \multicolumn{3}{|c|}{ Epoca lluviosa } \\
\hline & & \multicolumn{2}{|c|}{ IFC } & \multirow{2}{*}{ IPR } & \multicolumn{2}{|c|}{ IFC } & \multirow{2}{*}{ IPR } \\
\hline & & Especie & Familia & & Especie & Familia & \\
\hline \multicolumn{8}{|c|}{ Plantas vasculares angiospermas } \\
\hline \multirow[t]{2}{*}{ Aizoceae } & Glinus radiatus & 0,009091 & & & 0 & & \\
\hline & & & 0,009091 & $5,367 \mathrm{E}-05$ & & 0 & 0 \\
\hline \multirow[t]{2}{*}{ Alismataceae } & Echinodorus andrieuxii & 0,390909 & & & 0,32885 & & \\
\hline & & & 0,390909 & 0,00230775 & & 0,32885 & 0,001567 \\
\hline \multirow[t]{3}{*}{ Amaranthaceae } & Alternanthera sessilis & 0,045455 & & & 0,010204 & & \\
\hline & Amaranthus spinosus & 0,31 & & & 0,102041 & & \\
\hline & & & 0,355455 & 0,00209844 & & 0,112245 & 0,000535 \\
\hline \multirow[t]{2}{*}{ Apocynaceae } & Mesechites trifida & 0 & & & 0,040816 & & \\
\hline & & & 0 & 0 & & 0,040816 & 0,000194 \\
\hline \multirow[t]{2}{*}{ Araceae } & Pistia stratiotes & 0,054545 & & & 0,124768 & & \\
\hline & & & 0,054545 & 0,00032201 & & 0,124768 & 0,000594 \\
\hline \multirow[t]{3}{*}{ Asclepiadaceae } & Asclepias woodsoniana & 0,009091 & & & 0,020408 & & \\
\hline & Sarcostemma odoratum & 0,436364 & & & 0,112245 & & \\
\hline & & & 0,445455 & 0,00262976 & & 0,132653 & 0,000632 \\
\hline \multirow[t]{3}{*}{ Asteraceae } & Acmella oppositifolia & 0 & & & 0,5 & & \\
\hline & Ageratum conyzoides & 0,027273 & & & 0,204082 & & \\
\hline & Aster sp & 0 & & & 0,05102 & & \\
\hline
\end{tabular}


Continuación Cuadro 2.

\begin{tabular}{|c|c|c|c|c|c|c|c|}
\hline \multirow[t]{3}{*}{ Familia } & \multirow[t]{3}{*}{ Especie } & \multicolumn{3}{|c|}{ Eoca seca } & \multicolumn{3}{|c|}{ Epoca lluviosa } \\
\hline & & \multicolumn{2}{|c|}{ IFC } & \multirow[t]{2}{*}{ IPR } & \multicolumn{2}{|c|}{ IFC } & \multirow[t]{2}{*}{ IPR } \\
\hline & & Especie & Familia & & Especie & Familia & \\
\hline & Asteraceae & 0 & & & 0,102041 & & \\
\hline & Delilia biflora & 0 & & & 0,020408 & & \\
\hline & Eclipta alba & 2,056364 & & & 1,679267 & & \\
\hline & Emilia fosbergii & 0,072727 & & & 0,010204 & & \\
\hline & Erigeron annuus & 0 & & & 0,020408 & & \\
\hline & Isocarpha artriplicifolia & 0,018182 & & & 0 & & \\
\hline & Mikania micrantha & 0,090909 & & & 0,091837 & & \\
\hline & Pseudoelephantopus spicatu & is 0 & & & 0,030612 & & \\
\hline & Tridax procumbens & 0,581818 & & & 0,193878 & & \\
\hline & & & 2,847273 & 0,0168098 & & 2,903757 & 0,013835 \\
\hline \multirow[t]{3}{*}{ Boraginaceae } & Heliotropium fructicosum & 0,090909 & & & 0,438776 & & \\
\hline & Heliotropium indicum & 4,281818 & & & 1,346939 & & \\
\hline & & & 4,372727 & 0,02581455 & & 1,785714 & 0,008508 \\
\hline \multirow[t]{4}{*}{ Capparidaceae } & Cleome sp & 0 & & & 0,020408 & & \\
\hline & Cleome spinosa & 0,009091 & & & 0,010204 & & \\
\hline & Cleome viscosa & 0,009091 & & & 0,030612 & & \\
\hline & & & 0,018182 & 0,00010734 & & 0,061224 & 0,000292 \\
\hline \multirow[t]{2}{*}{ Combretaceae } & Combretum fructicosum & 0,018182 & & & 0 & & \\
\hline & & & 0,018182 & 0,00010734 & & 0 & 0 \\
\hline \multirow[t]{3}{*}{ Commelinaceae } & Commelina diffusa & 0,600909 & & & 4,255102 & & \\
\hline & Murdannia nodiflora & 0 & & & 4,665584 & & \\
\hline & & & 0,600909 & 0,00354749 & & 8,920686 & 0,042504 \\
\hline \multirow[t]{5}{*}{ Convolvulaceae } & Ipomoea purpurea & 0,063636 & & & 0,153061 & & \\
\hline & Iротоеа $\mathrm{sp}$ & 0,009091 & & & 0 & & \\
\hline & Merremia quinquefolia & 0,163636 & & & 0,020408 & & \\
\hline & Merremia umbellata & 0 & & & 0,010204 & & \\
\hline & & & 0,236364 & 0,00139538 & & 0,183673 & 0,000875 \\
\hline \multirow[t]{4}{*}{ Cucurbitaceae } & Cayaponia palustris & 0,127273 & & & 0,244898 & & \\
\hline & Cucumis melo & 0,209091 & & & 0,306122 & & \\
\hline & Momordica charanthia & 0 & & & 0,010204 & & \\
\hline & & & 0,336364 & 0,00198573 & & 0,561224 & 0,002674 \\
\hline \multirow[t]{11}{*}{ Cyperaceae } & Cyperus esculentus & 16,80364 & & & 17,71475 & & \\
\hline & Cyperus iria & 9,384545 & & & 10,83836 & & \\
\hline & Cyperus panamensis & 0 & & & 0,05102 & & \\
\hline & Cyperus rotundus & 0,8 & & & 1,650974 & & \\
\hline & Cyperus sp & 0,145455 & & & 0,011364 & & \\
\hline & Cyperus surinamensis & 0,018182 & & & 0,010204 & & \\
\hline & Cyperus tenerrimus & 0 & & & 0,010204 & & \\
\hline & Eleocharis elegans & 0,363636 & & & 0,021568 & & \\
\hline & Fimbristylis annua & 0 & & & 0,030612 & & \\
\hline & Fimbristylis littoralis & 1,50636 & & & 0,925325 & & \\
\hline & Hemicarpha micrantha & 0,018182 & & & 0,375928 & & \\
\hline
\end{tabular}


Continuación Cuadro 2.

\begin{tabular}{|c|c|c|c|c|c|c|c|}
\hline \multirow[t]{3}{*}{ Familia } & \multirow[t]{3}{*}{ Especie } & \multicolumn{3}{|c|}{ Epoca seca } & \multicolumn{3}{|c|}{ Epoca lluviosa } \\
\hline & & \multicolumn{2}{|c|}{ IFC } & \multirow[t]{2}{*}{ IPR } & \multicolumn{2}{|c|}{ IFC } & \multirow[t]{2}{*}{ IPR } \\
\hline & & Especie & Familia & & Especie & Familia & \\
\hline & Pycreus fugax & 0 & & & 0,010204 & & \\
\hline & Scleria pterota & 0,009091 & & & 1,336735 & & \\
\hline & Torulinium odoratum & 1,77 & & & 0,324443 & & \\
\hline & & & 30,81909 & 0,18194162 & & 33,31169 & 0,158717 \\
\hline \multirow[t]{9}{*}{ Euphorbiaceae } & Acalypha arvensis & 0 & & & 0,010204 & & \\
\hline & Caperonia palustris & 2,373636 & & & 6,712199 & & \\
\hline & Chamaesyce densiflora & 1,018182 & & & 0,388915 & & \\
\hline & Chamaesyce hirta & 0,018182 & & & 0,030612 & & \\
\hline & Chamaesyce hissopifolia & 0,181818 & & & 0,163265 & & \\
\hline & Croton lobulatus & 0,009091 & & & 0 & & \\
\hline & Phyllanthus niruri & 0,072727 & & & 0,153061 & & \\
\hline & Poinsettia heterophylla & 0 & & & 0,091837 & & \\
\hline & & & 3,673636 & 0,02168745 & & 7,550093 & 0,035973 \\
\hline \multirow[t]{21}{*}{ Fabaceae } & Acacia costarricensis & 0,009091 & & & 0 & & \\
\hline & Calopogonium mисопoides & 0,136364 & & & 0 & & \\
\hline & Chamaecrista hispitula & 0 & & & 0,010204 & & \\
\hline & Crotalaria pumila & 0,190909 & & & 0,122449 & & \\
\hline & Desmanthus virgatus & 0 & & & 0,020408 & & \\
\hline & Desmodium $\mathrm{sp}$ & 0 & & & 0,010204 & & \\
\hline & Desmodium scorpius & 0 & & & 0,010204 & & \\
\hline & Dioclea megacarpa & 0,009091 & & & 0 & & \\
\hline & Macroptilium lathyroides & 0,090909 & & & 0,081633 & & \\
\hline & Mimosa pigra & 0,127273 & & & 0,081633 & & \\
\hline & Mimosa pudica & 0,018182 & & & 0,010204 & & \\
\hline & Phaseolus lunatus & 0,009091 & & & 0 & & \\
\hline & Rhynchosia minima & 0,009091 & & & 0,132653 & & \\
\hline & Sesbania emerus & 0,056364 & & & 0,032931 & & \\
\hline & Sesbania exaltata & 0,009091 & & & 0 & & \\
\hline & Stylosanthes guayanensis & 0 & & & 0,010204 & & \\
\hline & Tephrosia sp & 0 & & & 0,010204 & & \\
\hline & Teramnus volubilis & 0,018182 & & & 0,020408 & & \\
\hline & Vigna sp & 0 & & & 0,020408 & & \\
\hline & Vigna linearis & 0,981818 & & & 6,307282 & & \\
\hline & & & 1,665455 & 0,00983207 & & 6,88103 & 0,032785 \\
\hline \multirow[t]{2}{*}{ Gentianaceae } & Centaurium quitense & 0,027273 & & & 0 & & \\
\hline & & & 0,027273 & 0,00016101 & & 0 & 0 \\
\hline \multirow[t]{2}{*}{ Hidrocaritaceae } & Hydrilla verticillata & 13,03636 & & & 11,91837 & & \\
\hline & & & 13,03636 & 0,07696064 & & 11,91837 & 0,056786 \\
\hline \multirow[t]{2}{*}{ Labiatae } & Hyptis recurvata & 0,227273 & & & 0 & & \\
\hline & & & 0,227273 & 0,00134171 & & 0 & 0 \\
\hline \multirow[t]{2}{*}{ Limnocharitaceae } & Limnocharis flava & 0,181818 & & & 0,256725 & & \\
\hline & & & 0,181818 & 0,00107337 & & 0,256725 & 0,001223 \\
\hline
\end{tabular}


Continuación Cuadro 2.

\begin{tabular}{|c|c|c|c|c|c|c|c|}
\hline \multirow[t]{3}{*}{ Familia } & \multirow[t]{3}{*}{ Especie } & \multicolumn{3}{|c|}{ Epoca seca } & \multicolumn{3}{|c|}{ Epoca lluviosa } \\
\hline & & \multicolumn{2}{|c|}{ IFC } & \multirow[t]{2}{*}{ IPR } & \multicolumn{2}{|c|}{ IFC } & \multirow[t]{2}{*}{ IPR } \\
\hline & & Especie & Familia & & Especie & Familia & \\
\hline \multirow[t]{2}{*}{ Loganiaceae } & Spigelia anthelmia & 0 & & & 0,010204 & & \\
\hline & & & 0 & 0 & & 0,010204 & 4,9E-05 \\
\hline \multirow[t]{2}{*}{ Lythraceae } & Ammannia coccinea & 0,081818 & & & 0,129406 & & \\
\hline & & & 0,081818 & 0,00048302 & & 0,129406 & 0,000617 \\
\hline \multirow[t]{7}{*}{ Malvaceae } & Malachra capitata & 0,546364 & & & 1,1032 & & \\
\hline & Malachra radiata & 0,009091 & & & 0,010204 & & \\
\hline & Malvaviscus arboreus & 0 & & & 0,010204 & & \\
\hline & Pavonia cancellata & 0 & & & 0,020408 & & \\
\hline & Peltaea sessiliflora & 0,009091 & & & 0,091837 & & \\
\hline & Sida acuta & 0,1 & & & 0,040816 & & \\
\hline & & & 0,664545 & 0,00392317 & & 1,27667 & 0,006083 \\
\hline \multirow[t]{2}{*}{ Marantaceae } & Thalia geniculata & 0,118182 & & & 1,361549 & & \\
\hline & & & 0,118182 & 0,00069769 & & 1,361549 & 0,006487 \\
\hline \multirow[t]{2}{*}{ Marsileaceae } & Marsilea diflexa & 0,018182 & & & 0 & & \\
\hline & & & 0,018182 & 0,00010734 & & 0 & 0 \\
\hline \multirow[t]{2}{*}{ Molluginaceae } & Mollugo verticillata & 0,090909 & & & 0,010204 & & \\
\hline & & & 0,090909 & 0,00053669 & & 0,010204 & 4,9E-05 \\
\hline \multirow[t]{2}{*}{ Moraceae } & Dorstenia drakena & 0,027273 & & & 0,030612 & & \\
\hline & & & 0,027273 & 0,00016101 & & 0,030612 & 0,000146 \\
\hline \multirow[t]{2}{*}{ Nymphaeaceae } & Nymphaea ampla & 1,509091 & & & 1,017161 & & \\
\hline & & & 1,509091 & 0,00890897 & & 1,017161 & 0,004846 \\
\hline \multirow[t]{3}{*}{ Onagraceae } & Ludwigia aff-palustris & 0,036364 & & & 0,164657 & & \\
\hline & Ludwigia decurrens & 0,946364 & & & 0,987709 & & \\
\hline & & & 0,982727 & 0,00580157 & & 1,152365 & 0,005491 \\
\hline \multirow[t]{2}{*}{ Passifloraceae } & Passiflora foetida & 1,5 & & & 0,397959 & & \\
\hline & & & 1,5 & 0,0088553 & & 0,397959 & 0,001896 \\
\hline \multirow[t]{2}{*}{ Phytolacaceae } & Rivina humilis & 0,009091 & & & 0 & & \\
\hline & & & 0,009091 & 5,367E-05 & & 0 & 0 \\
\hline \multirow[t]{10}{*}{ Poaceae } & Anthephora hermaphrodita & 1,254545 & & & 2,816327 & & \\
\hline & Brachiaria fasciculata & 0,009091 & & & 0,459184 & & \\
\hline & Brachiaria mollis & 0 & & & 0,020408 & & \\
\hline & Brachiaria mutica & 2,209091 & & & 5,249536 & & \\
\hline & Cenchrus pilosus & 0,081818 & & & 0,030612 & & \\
\hline & Chloris barbata & 0 & & & 0,010204 & & \\
\hline & Cynodon dactylon & 2,409091 & & & 1,520408 & & \\
\hline & Dactyloctenium aegyptium & 0 & & & 0,030612 & & \\
\hline & Digitaria sanguinalis & 0,518182 & & & 1,540816 & & \\
\hline & Dichanthelium aristatum & 0,136364 & & & 0 & & \\
\hline
\end{tabular}


Continuación Cuadro 2.

\begin{tabular}{|c|c|c|c|c|c|c|c|}
\hline \multirow[t]{3}{*}{ Familia } & \multirow[t]{3}{*}{ Especie } & \multicolumn{3}{|c|}{ Epoca seca } & \multicolumn{3}{|c|}{ Epoca lluviosa } \\
\hline & & \multicolumn{2}{|c|}{ IFC } & \multirow[t]{2}{*}{ IPR } & \multicolumn{2}{|c|}{ IFC } & \multirow[t]{2}{*}{ IPR } \\
\hline & & Especie & Familia & & Especie & Familia & \\
\hline & Echinochloa colona & 17,78 & & & 24,6199 & & \\
\hline & Echinochloa polystachya & 2,036364 & & & 0,030612 & & \\
\hline & Eleusine indica & 0,027273 & & & 0,020408 & & \\
\hline & Eragrostis ciliaris & 0,145455 & & & 0,091837 & & \\
\hline & Eragrostis glomerata & 1,245455 & & & 0 & & \\
\hline & Eragrostis pectinacea & 0,027273 & & & 0,081633 & & \\
\hline & Eragrostis pilosa & 0,009091 & & & 0,010204 & & \\
\hline & Hymenachne amplexicaulis & 0,981818 & & & 1,511364 & & \\
\hline & Hyparrhenia rufa & 3,427273 & & & 0,387755 & & \\
\hline & Ischaemum rugosum & 0,009091 & & & 0,571429 & & \\
\hline & Ixophorus unisetus & 12,84545 & & & 24,88103 & & \\
\hline & Leersia hexandra & 2,254545 & & & 1,622449 & & \\
\hline & Leptochloa filiformis & 0,081818 & & & 0,27551 & & \\
\hline & Leptochloa scabra & 2,236364 & & & 0,451299 & & \\
\hline & Luziola subintegra & 0 & & & 0,795918 & & \\
\hline & Oplismenus burmannii & 0,018182 & & & 0,602041 & & \\
\hline & Oryza latifolia & 1,177273 & & & 0,378942 & & \\
\hline & Oryza rufipogon & 8,474545 & & & 8,314703 & & \\
\hline & Oryza sativa(voluntario) & 19,12091 & & & 17,23864 & & \\
\hline & Panicum capillare & 0,345455 & & & 0,460343 & & \\
\hline & Panicum laxum & 0,036364 & & & 0,010204 & & \\
\hline & Panicum maximum & 4,172727 & & & 3,408163 & & \\
\hline & Panicum trichoides & 0,009091 & & & 0,112245 & & \\
\hline & Paspalum conjugatum & 0 & & & 0,010204 & & \\
\hline & Paspalum fluitans & 1,481818 & & & 2,026206 & & \\
\hline & Paspalum urvillei & 0,009091 & & & 0 & & \\
\hline & Paspalum virgatum & 0 & & & 0,010204 & & \\
\hline & Rottboellia exaltata & 13,50909 & & & 18,21429 & & \\
\hline & Setaria geniculata & 0,009091 & & & 0 & & \\
\hline & & & 98,08909 & 0,5790725 & & 117,8156 & 0,561345 \\
\hline \multirow[t]{3}{*}{ Pontederiaceae } & Heteranthera limosa & 0,363636 & & & 4,687616 & & \\
\hline & Heteranthera reniformis & 0,081818 & & & 0,578386 & & \\
\hline & & & 0,445455 & 0,00262976 & & 5,266002 & 0,02509 \\
\hline \multirow[t]{2}{*}{ Portulacaceae } & Portulaca oleracea & 0,200909 & & & 0,183673 & & \\
\hline & & & 0,200909 & 0,00118607 & & 0,183673 & 0,000875 \\
\hline \multirow[t]{4}{*}{ Rubiaceae } & Richardia scabra & 0 & & & 0,010204 & & \\
\hline & Spermacoce gracilis & 0,027273 & & & 0,020408 & & \\
\hline & Spermacoce latifolia & 0 & & & 0,27551 & & \\
\hline & & & 0,027273 & 0,00016101 & & 0,306122 & 0,001459 \\
\hline \multirow[t]{2}{*}{ Salvinaceae } & Azolla mexicana & 0,690909 & & & 0,020408 & & \\
\hline & & & 0,690909 & 0,00407881 & & 0,020408 & $9,7 \mathrm{E}-05$ \\
\hline \multirow[t]{2}{*}{ Sapindaceae } & Paullinia sp & 0,590909 & & & 0,173469 & & \\
\hline & & & 0,590909 & 0,00348845 & & 0,173469 & 0,000827 \\
\hline
\end{tabular}


Continuación Cuadro 2.

\begin{tabular}{|c|c|c|c|c|c|c|c|}
\hline \multirow[t]{3}{*}{ Familia } & \multirow[t]{3}{*}{ Especie } & \multicolumn{3}{|c|}{ EPOCA SECA } & \multicolumn{3}{|c|}{ EPOCA LLUVIOSA } \\
\hline & & \multicolumn{2}{|c|}{ IFC } & \multirow[t]{2}{*}{ IPR } & \multicolumn{2}{|c|}{ IFC } & \multirow[t]{2}{*}{ IPR } \\
\hline & & Especie & Familia & & Especie & Familia & \\
\hline \multirow[t]{5}{*}{ Scrophulariaceae } & Bacopa repens & 0,081818 & & & 2,714286 & & \\
\hline & Mecardonia procumbens & 0,163636 & & & 0,204082 & & \\
\hline & Scoparia dulcis & 0,009091 & & & 0 & & \\
\hline & Stemodia durantifolia & 2,909091 & & & 0,849258 & & \\
\hline & & & 3,163636 & 0,01867664 & & 3,767625 & 0,017951 \\
\hline \multirow[t]{6}{*}{ Solanaceae } & Lycianthes $\mathrm{sp}$ & 0,427273 & & & 0,602041 & & \\
\hline & Physalis minuta & 0 & & & 0,020408 & & \\
\hline & Physalis sp & 0 & & & 0,010204 & & \\
\hline & Solanum americanum & 0,063636 & & & 0,061224 & & \\
\hline & Solanum ochraceo-ferrugin & $n 0$ & & & 0,010204 & & \\
\hline & & & 0,490909 & 0,0028981 & & 0,704082 & 0,003355 \\
\hline \multirow[t]{3}{*}{ Sterculiaceae } & Melochia nodiflora & 0,009091 & & & 0,020408 & & \\
\hline & Melochia pyramidata & 0,5 & & & 0,020408 & & \\
\hline & & & 0,509091 & 0,00300544 & & 0,040816 & 0,000194 \\
\hline \multirow[t]{2}{*}{ Tiliaceae } & Corchorus siliquosus & 0,290909 & & & 0,397959 & & \\
\hline & & & 0,290909 & 0,0017173 & & 0,397959 & 0,001896 \\
\hline \multirow[t]{2}{*}{ Typhaceae } & Typha angustifolia & 0 & & & 0,010204 & & \\
\hline & & & 0 & 0 & & 0,010204 & $4,9 \mathrm{E}-05$ \\
\hline \multirow[t]{3}{*}{ Verbenaceae } & Priva lappulacea & 0,009091 & & & 0,030612 & & \\
\hline & Stachytarpheta jamaicensis & 0,036364 & & & 0,154221 & & \\
\hline & & & 0,045455 & 0,00026833 & & 0,184833 & 0,000881 \\
\hline \multirow[t]{2}{*}{ Vitaceae } & Cissus pseudosicyoides & 0,418182 & & & 0,091837 & & \\
\hline & & & 0,418182 & 0,00246862 & & 0,091833 & 0,000438 \\
\hline \multirow[t]{2}{*}{ Zigophyllaceae } & Kallstroemia pubescens & 0,081818 & & & 0,428571 & & \\
\hline & & & 0,081818 & 0,00048299 & & 0,428571 & 0,002042 \\
\hline \multirow[t]{3}{*}{ No identificada } & No identificada & 0 & & & 0,010204 & & \\
\hline & & & 0 & 0 & & 0,010204 & 4,9E-05 \\
\hline & Subtotal de angiospermas & & 169,3627 & 0,99978587 & & 209,8611 & 0,999905 \\
\hline \multicolumn{8}{|c|}{ Plantas no vasculares y Pterodophytas } \\
\hline \multirow[t]{2}{*}{ Pterodophyta } & Salpichlaena sp & 0,027273 & & & 0,020408 & & \\
\hline & & & 0,027273 & 0,000161 & & 0,020408 & $9,7 \mathrm{E}-05$ \\
\hline \multirow[t]{4}{*}{ Selaginellaceae } & Selaginella sp. & 0,009091 & & & 0 & & \\
\hline & & & 0,009091 & $5,367 \mathrm{E}-05$ & & 0 & 0 \\
\hline & $\begin{array}{l}\text { Subtotal de no vasculares } \\
\text { y helechos }\end{array}$ & & 0,036364 & 0,00021466 & & 0,020408 & $9,7 \mathrm{E}-05$ \\
\hline & Total de todo el inventario & & 169,3991 & 1 & 209,8815 & 1 & \\
\hline
\end{tabular}


diferencia de las poáceas y ciperáceas, representadas por varias especies.

Se presentó una disminución en el IPR de las familias Boraginaceae y Asteraceae en la época lluviosa, con respecto al IPR de la época seca, lo cual indica que los miembros de estas familias fueron desplazados en importancia por especies de otras familias. En el caso de las boraginaceas, esta disminución del IPR se debió a una reducción del IFC de Heliotropium indicum, lo cual parece indicar que esta especie no se adapta bien a las condiciones de la época lluviosa. En el caso de las Asteraceae, los IFC de ambas épocas se mantuvieron muy parecidos, por lo que la disminución del IPR sólo se explica por un aumento en la participación de otras familias en la época lluviosa.

Se observó un comportamiento inverso entre el grupo compuesto por las familias Boraginaceae y Asteraceae y el grupo de especies de las familias Fabaceae y Commelinaceae (Figura 1). Mientras que el primer grupo fue mayor en IPR en la época seca en comparación con el segundo grupo, al establecerse la época lluviosa este último alcanzó IPR más altos que los de las dos primeras familias. Esto puede deberse a varios hechos, competencia entre los individuos de esas familias, el clima, el programa de manejo de malezas o la combinación de varios factores, que desfavorecieron a los miembros de uno de los dos grupos de familias y los espacios vacíos creados fueron ocupados por los miembros del otro grupo. Esto es importante desde un punto de vista de manejo ya que se pueden favorecer a aquellas familias que presentan especies de porte bajo (Fabaceae y Commelinaceae) para que sirvan de cobertura de los canales y controlen el establecimiento de especies de mayor porte y posiblemente más competitivas de otras familias.

Con poáceas se observó variación entre las épocas seca y lluviosa a nivel de las especies más importantes. $E$. colonum pasó a ser la segunda especie en importancia en la berna, en la época lluviosa, mientras que en la época seca no estuvo dentro de las tres primeras. La mayor humedad en el suelo favoreció a esta especie por lo que desplazó a otras. Algo similar sucedió con $R$. exaltata que pasó a ocupar el tercer lugar en importancia. A nivel de talud interior se dió un desplazamiento de $R$. exaltata en la época seca como especie más importante por E. colonum en la época lluviosa. $I$. unisetus desplazó a Oryza sativa ( arroz voluntario ) del primer lugar en el talud exterior al establecerse la época lluviosa. Este hecho y el que en la época seca I. unisetus fuera la más importante en la berna son indicios de una gran plasticidad de esta especie en comparación con

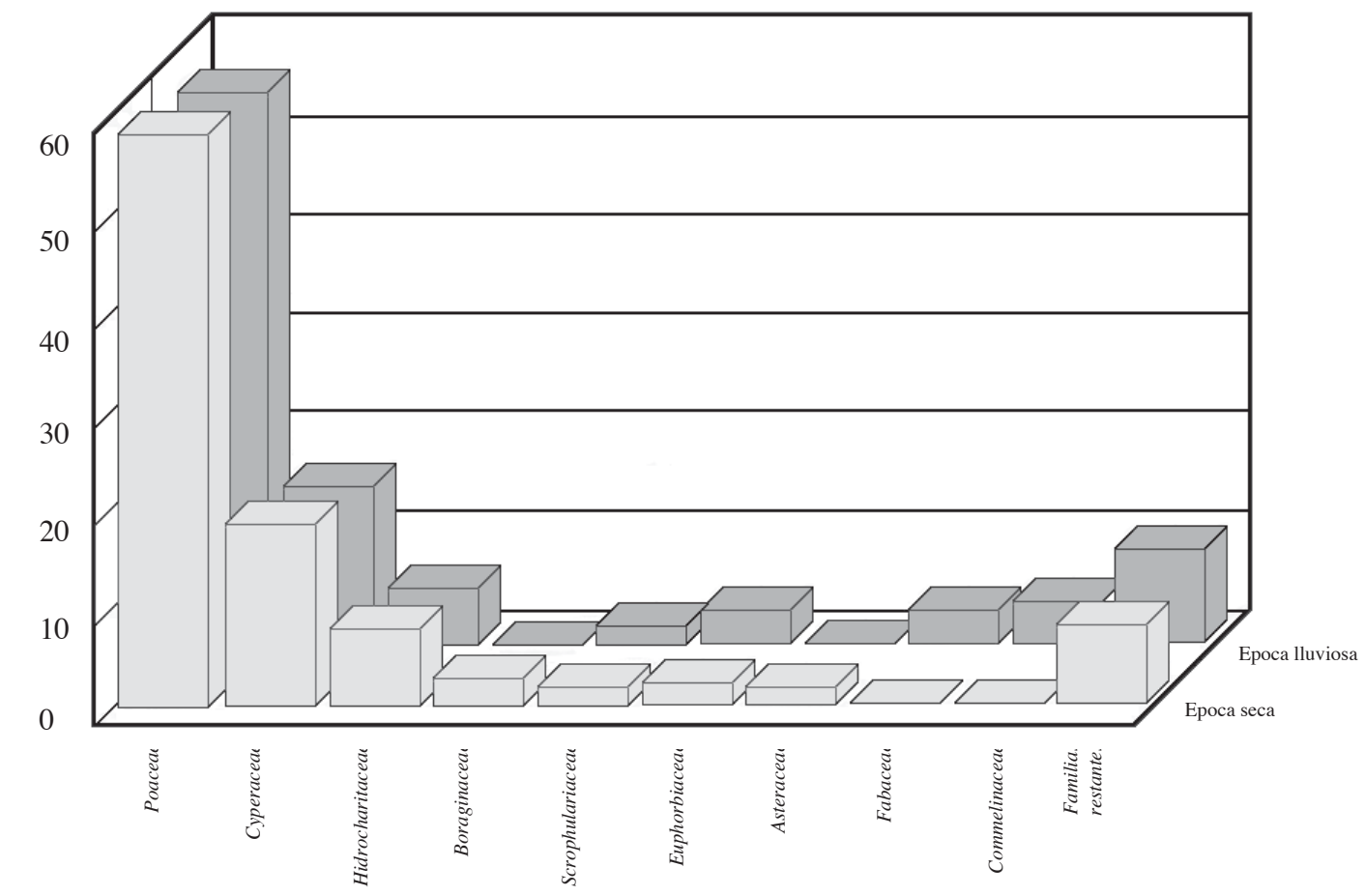

Fig. 1. Indice de participación relativo de las principales familias de malezas en las dos épocas climáticas. Finca El Cerrito, Liberia. Guanacaste. 1992. 
otras. En la época seca se observó que las dos especies más importantes en el plan, también lo fueron en el cultivo, lo que es una muestra de la estrecha relación entre estos dos hábitats. Una situación similar se presentó en la época lluviosa con la especie más importante en estos dos hábitats. Esto confirma la necesidad de un buen control de malezas en el plano del canal para reducir la incidencia en el cultivo.

Un mejor control de E. colonum en la época lluviosa podría explicar que no sea la principal maleza en el plan y en el cultivo. La presencia de lotes en barbecho fue la razón de la presencia de $O$. sativa (arroz voluntario) como especie importante en el cultivo en las dos épocas.

En las ciperaceae no se dio variación entre las especies más importantes en ambas épocas.

En las latifoliadas hubo variación en cuanto a las especies más importantes por hábitat. Mientras que $H$. indicum fue la única especie de este grupo que se le consideró importante en la época seca en la berna, en la

Cuadro 3. Principales especies de Poaceae, Cyperaceae y latifoliadas en cada hábitat del canal. Epocas seca y lluviosa. Finca El Cerrito, Liberia. Guanacaste, Costa Rica. 1992.

\begin{tabular}{llllll}
\hline & Berna & T. interior & T. exterior & Plan & Cultivo \\
\hline Poaceas Epoca seca & & & & & \\
& I. unisetus & R. exaltata & O.sativa (v) & E. colona & E.colona \\
& P. maximum & E. colona & I.unisetus & O. sativa (v) & O.sativa (v) \\
& O.sativa (v) & O. sativa (v) & R. exaltata & O.rufipogon & O.latifolia \\
& & I.unisetus & E.colona & P.fluitans & \\
& & O.rufipogon & C.dactylon & &
\end{tabular}

Poaceae Epoca lluviosa

$\begin{array}{lll}\text { I.unisetus } & \text { E. colona } & \text { I. unisetus } \\ \text { E. colona } & \text { O. sativa }(v) & \text { O.sativa }(v) \\ \text { R. exaltata } & \text { I.unisetus } & \text { E. colona } \\ \text { P. maximum } & \text { O.rufipogon } & \text { R. exaltata } \\ \text { B. mutica } & \text { R. exaltata } & \\ & \text { B. mutica } & \end{array}$

Cyperaceae Epoca seca
C. esculentus
C. esculentus
C. esculentus
C.iria.
C. esculentus
C.iria
F. littoralis
C. iria

Cyperaceae Epoca lluviosa
C. esculentus
C. esculentus
C. esculentus
IFC muy bajos
C. iria
C. iria
C. iria
C. iria

Latifoliadas Epoca seca

$\begin{array}{ll}\text { H. indicum } & \text { S. durantifolia } \\ & \text { H. indicum } \\ & \text { C. palustris } \\ & \text { E. alba }\end{array}$

IFC muy bajos

H. verticillata

N. ampla

IFC muy bajos

Latifoliadas Epoca lluviosa
$V$. linearis
M. nodiflora
V. linearis
H. verticillata
H. limosa
C. palustris
C. palustris
C. palustris
B. repens
C. diffusa
C. diffusa
C. diffusa
M. nodiflora
$V$. linearis
E. alba

$\mathrm{v}=$ voluntario 
época lluviosa se presentaron cuatro especies que desplazaron a H. indicum. En el talud interior de cuatro especies importantes en la época seca, dos se mantuvieron dentro de este grupo para la época lluviosa. Una de las desplazadas fue $H$. indicum. Vigna linearis una leguminosa presente en la época seca en menor magnitud se volvió importante en la época lluviosa en la berna y los taludes. A nivel de plan no hubo variaciones importantes. En el cultivo las dos especies importantes que aparecieron en la época lluviosa no lo fueron en la época seca. En el caso de Heteranthera limosa es posible que su presencia está asociada con el fangueo que se realizó para la siembra de invierno, ya que esta labor crea condiciones apropiadas para especies acuáticas.

\section{CONCLUSIONES}

La mayor parte de las especies registradas se localizaron en el canal de riego y una menor cantidad en la zona de cultivo. Esto significa que la zona del cultivo fue más homogénea que el resto de hábitats del canal. Dentro del canal, el plan fue la sección más homogénea. El manejo que recibe el cultivo y la presencia de una lámina de agua son las principales causas de esa homogeneidad ya que reducen el número de especies que se pueden adaptar a dichas condiciones. De acuerdo a esto, los hábitats muestreados se pueden agrupar en tres categorías por la similitud de la población de malezas. Un primer grupo lo constituyen la berna y los taludes interior y exterior; el segundo, el plan del canal y el tercero la zona del cultivo. En el primer caso si bien existieron ciertas especies que predominaron ya sea en la berna o en alguno de los taludes, las diferencias entre ellos no fueron tan grandes y muchas especies se localizaron en los tres hábitats indistintamente. El plano del canal se diferencia del anterior grupo y tiende a presentar especies más adaptadas a la presencia de agua, sin embargo algunas especies presentes en los hábitats del anterior grupo también estuvieron en el plano del canal. Por su parte la zona de cultivo presentó un menor número de especies, por razones obvias de un manejo más esmerado de malezas.

La cobertura de malezas sufrió un incremento al pasar de la época seca a la lluviosa, producto de la mayor humedad en el suelo. Sin embargo no todas las especies incrementaron su IFC al pasar a la época lluviosa. Por otro lado, diversas especies sufrieron reducción de su IPR a pesar de que incrementaron su IFC, esto debido a que en el muestreo total otras especies experimentaron mayores incrementos.

De las especies registradas la mayoría presentaron IFC muy bajos, mientras que una menor cantidad de especies acumuló la mayor parte del IFC de una región.

La familia más importante de acuerdo al I.P.R. fue la Poaceae, le siguió en importancia la Cyperaceae. El grupo de familias latifoliadas terrestres y acuáticas a pesar de ser el más numeroso en especies no presentó valores de IPR semejantes a los de las dos anteriores familias. Sin embargo, debe aclararse que se presentaron especies del grupo de latifoliadas y acuáticas, que por su IFC y su IPR, se catalogaron como importantes.

Una de las especies que más adaptabilidad presentó y apareció en todos los hábitats fue Echinochloa colonum. Es importante poner más atención a esta especie ya que constituye una maleza importante del cultivo y aunque es controlada dentro del área de siembra, ella se está reproduciendo en el canal y es introducida al cultivo con el agua de riego. Similar situación se presentó con las especies O. rufipogon ( Arroz rojo), O. latifolia (Arroz pato), C. iria y varias latifoliadas.

\section{LITERATURA CONSULTADA}

SANCHEZ, V.M. 1993 Caracterización de la vegetación silvestre circundante y asociada al cultivo del melón (Cucumis melo L.) e identificación de especies hospederas de los principales virus que lo infectan y sus vectores. Tesis de Licenciatura. Escuela de Ciencias Biológicas. Universidad Nacional. Heredia, Costa Rica. 92 p.

SOTO, A.; AGÜERO, R. 1992. Combate químico de malezas en arroz. San José, Costa Rica, Editorial Universidad de Costa Rica. 81 p.

THOMAS, A.G. 1991. Floristic composition and relative abundance of weeds in annual crops. Weed Science. 33 (1): $34-43$. 